\title{
Menkes Disease
}

National Institute of Neurological Disorders and Stroke (NINDS)

\section{Source}

National Institute of Neurological Disorders and Stroke (NINDS). Menkes Disease

Information Page.

Menkes disease is caused by a defective gene named ATPTA1 that regulates the metabolism of copper in the body. The disease primarily affects male infants. Copper accumulates at abnormally low levels in the liver and brain, but at higher than normal levels in the kidney and intestinal lining. Affected infants may be born prematurely, but appear healthy at birth and develop normally for 6 to 8 weeks. Then symptoms begin, including floppy muscle tone, seizures, and failure to thrive. Menkes disease is also characterized by subnormal body temperature and strikingly peculiar hair, which is kinky, colorless or steel-colored, and breaks easily. There is often extensive neurodegeneration in the gray matter of the brain. Arteries in the brain may be twisted with frayed and split inner walls. This can lead to rupture or blockage of the arteries. Weakened bones (osteoporosis) may result in fractures. 